\title{
Web based Multi Product Inventory Optimization using Genetic Algorithm
}

\author{
Priya P \\ Research Scholar, \\ Dept of computer science, Bharathiar \\ University, Coimbatore
}

\author{
Dr.K.lyakutti \\ Senior Professor, Madurai Kamarajar \\ University, Madurai
}

\begin{abstract}
This paper presents an approach to optimize the reorder level (ROL) in the manufacturing unit taking consideration of the stock levels at the factory and the distribution centers of the supply chain, which in turn helps the production unit to optimize the production level and minimizing the inventory holding cost. Genetic algorithm is used for the optimization in a multi product, multi level supply chain in a web enabled environment. This prediction of optimal ROL enables the manufacturing unit to overcome the excess/ shortage of stock levels in the upcoming period.
\end{abstract}

\section{INTRODUCTION}

Inventory encompasses all raw materials, work in process, and finished goods within the supply chain. Changing Inventory policies can dramatically alter the supply chain's efficiency and responsiveness. Inventory is an important cross functional driver of supply chain performance. An important role that can be satisfied by having the product ready and available when the customer wants it. Inventory is held throughout the supply chain in the form of raw materials, work in progress, and finished goods.

\section{LITERATURE REVIEW}

Chandra et al (2010) has developed a two-warehouse inventory model for deteriorating items when demand is price sensitive. In this article, an attempt has been made to develop an order-level inventory model for deteriorating items with two-storage facility under a bulk release pattern. They have assumed that the deterioration rate of the items stored might be different in the two ware-houses due to the difference in the environmental conditions or preserving conditions. Also the same model is applicable when the deterioration rate is same in both the warehouse. A computational procedure is also proposed to obtain the optimal number of shipments, optimal shipment quantity and optimal selling price, which maximizes the total profit of the system.

Narmadha, et al (2010), proposed a Genetic Algorithm with uniform cross over to study the stock level that needs essential inventory management control. The authors discussed that the proposed approach of inventory management satisfied the objectives such as minimization of the total supply chain cost and determination of the products due to which the supplier endured either additional holding cost or shortage cost. The authors also proposed that the complexity increases when more distribution centers, agents and multi products are involved in the supply chain. Following the predicted stock levels excess storage/ shortage inventory levels can be avoided in the upcoming period and hence the increase in the supply chain cost can be avoided.

Radhakrishnan, et al (2010) proposed a novel approach based on Genetic Algorithm to predict the most emerging stock levels of the future by considering the stock levels of the past periods. They have discussed the supply chain where multiple factories, multiple products and multiple agents are the members of the supply chain. The inventory optimization in the supply chain is distinctively determined to achieve minimum total supply chain cost using the proposed approach.

Kannan et al (2009), presents an integrated forward logistics multi-echelon distribution inventory supply chain model and closed loop multi-echelon distribution inventory supply chain model for built to order environment using genetic algorithm and particle swarm optimization. They have developed a new model that integrates the forward and reverse logistics in a built to order supply chain environment. This model is used to optimize the distribution and inventory in the supply chain using Genetic Algorithm to minimize the total supply chain cost, which includes purchasing, production, distribution and inventory related costs to the build to order environment.

Xiuli Chao and Sean X. Zhou (2009), has presented an efficient algorithm for computing the optimal control parameters in inventory control policy. An optimal ordering policy for the multi echelon system with both batch ordering and fixed replenishment intervals, and also a distribution function solution for the optimal reorder points are given solely in terms of lead lime demand distribution function. They had shown that the optimal average cost is minimized when all stages are synchronized, hence the optimal reorder point for each stage is lowest as all the stages are synchronized.

Maity et al (2009), has proposed optimum production for a multi-item production inventory system with deteriorating units, space capacity constraint. They formulated a multi item system with a resource constraint via optimal control theory for steady state case and transitive case respectively. In future the formulation and analysis can be extended to other production inventory problem in fuzzy, fuzzystochastic environment with different types of demand, defectiveness and deterioration. 
Uthayakumar et al (2009), has proposed an optimal replenishment policy by considering stock dependent consumption rate for non instantaneous deteriorating items with money inflation and time discounting. The authors have framed deterministic inventory model for noninstantaneous deteriorating items with stock dependent consumption. Shortages are allowed and partially backlogged. The aim is to minimize the retailer's total inventory cost by considering effect of inflation and time value of money.

Jianhui Wang et al (2006), proposed an improved Genetic Algorithm for the spare part store management. The objective is to optimize the number of stores and reduce the cost of store management. The improved genetic algorithm namely Genetic algorithm of Deuce Symmetrical Crisscross Based on Real Value (GADSC) operates on real number bound. The optimum value of the spare part stores is the real value. The authors constructed a multi objective optimal model and single period spare part store management optimal model. The authors concluded that the proposed improved methodology will aim in minimizing the management cost and interrelated expenses.

Gunasekaran, et al (2004), proposed a framework to promote a better understanding of the importance of supply chain performance measures and metrics. Based on selected literatures on supply chain performance the framework was designed. He designed a seven page questionnaire divided into four categories addressing basic four activities of supply chain. The performance measures were categorized based on importance, highly, moderately and less.

Jeffery Jones, et al (2002) discussed a Genetic algorithm approach for sourcing decisions within the supply chain. The authors proposed a simulation technique in the optimization process for a multi objective problem. Instead of a analytical evaluation, each solution was simulated to determine its performance.
From the literature study on optimizing the ROL of inventory in a multi product environment, it is clear that a web based attempt to monitor the stock levels across the

supply chain from the manufacturing unit through the distributors and online optimization of the ROL using genetic algorithm has not been reported in the literature.

\subsection{Steps in Genetic Algorithm}

Step1 Choose a coding to represent problem parameters, a selection operator, crossover operator, and a mutation operator. Choose population size $n$, crossover probability $\left(\mathrm{P}_{\mathrm{c}}\right)$ and mutation probability, $\left(\mathrm{P}_{\mathrm{m}}\right)$. Initialize a random population of strings of size (I). Choose a maximum allowable generation number $\mathrm{t}_{\max }$, set $\mathrm{t}=0$.

Step 2 Evaluate each string in the population.

Step 3 If $t>t_{\max }$, or other termination criteria is satisfied, Terminate.

Step 4 Perform reproduction on the population.

Step 5 Perform crossover on random pair of strings

Step 6 Perform mutation on every string

Step 7 Evaluate strings in the new population, set $\mathrm{t}=\mathrm{t}+1$ and go to step 3

The algorithm is straight forward with repeated application of three operators (step 4 to step 7) to a population of points.

\section{SYSTEM DESIGN}

In the proposed system shown in Figure 1, the sales of multiple products are centralized with direct interface between retailer and manufacturer. The reorder level (ROL) of the inventory is optimized using genetic algorithm in the factory. A centralized sale helps to monitor the sales of the products throughout the supply chain with visibility.

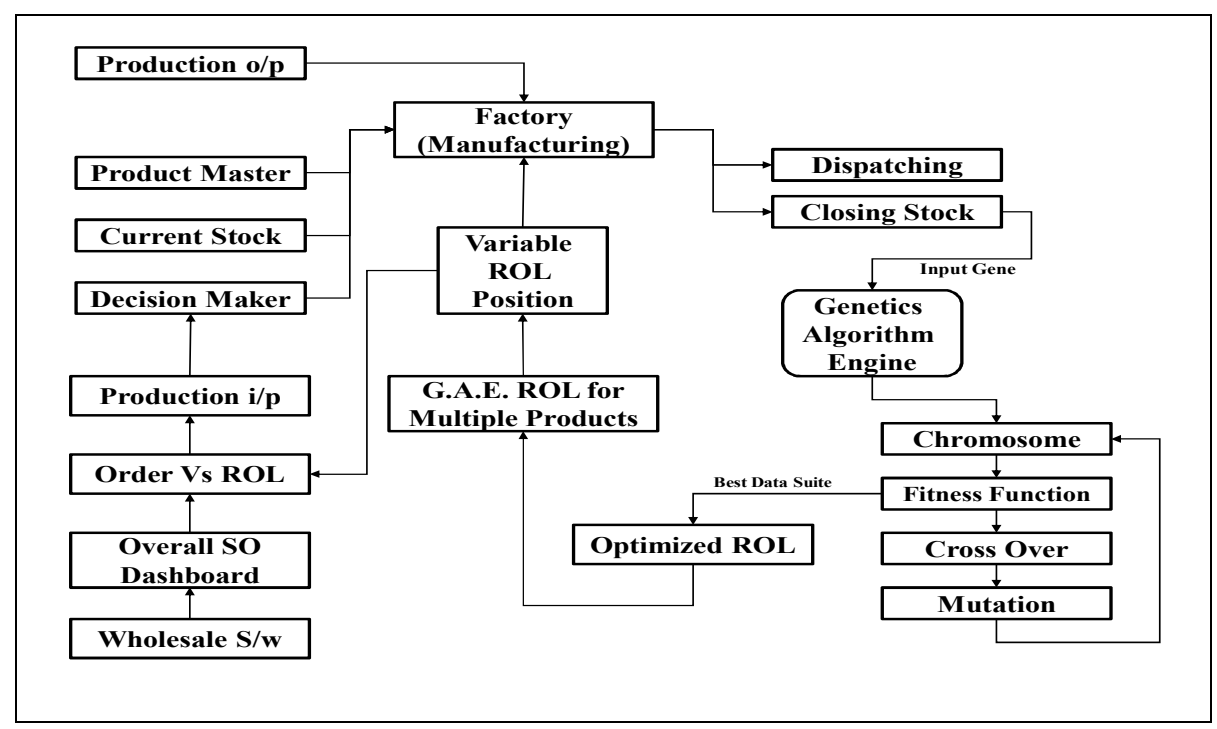

Figure 1 System design 
The two stages of the supply chain namely the Manufacturing industry and the distribution centers are considered in the study. Our exemplary supply chain consists of Manufacturing unit, Distribution center 1 and distribution center 2 , thus a three level supply chain. The retailer's stock level is combined with the distributor. We consider the stock level for 52 week, i.e., for one year. If a new entry is given that will be taken as a fifty second entry and the first entry will move off (stack, last in first out). The objective of the problem is to obtain the near optimal reorder level in the manufacturing unit, which helps the production unit to take decision about the production quantity. The a novel approach based on genetic algorithm is used to predict the optimal reorder level that contributes to reduce the inventory holding cost.

\section{(i) Chromosome Representation}

The weekly stock level of the Manufacturing unit, Distribution centers 1 and 2 are taken as a gene of the chromosome. Here we are using only three members of the supply chain, hence the length of the supply chain is three. Initially two chromosomes are generated and GA operations are performed in the GA Engine (GAE).

\section{Chromosome I}

Chromosome II

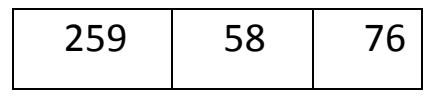

\begin{tabular}{|l|l|l|}
\hline 351 & 72 & 43 \\
\hline
\end{tabular}

A single point crossover operator is used as illustrated below.

Before Cross-over

Chromosome I

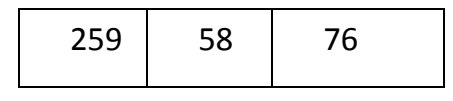

Chromosome II

After Cross-over

Chromosome I

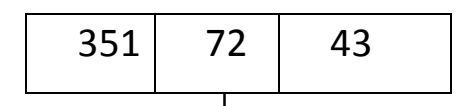

Chromosome II
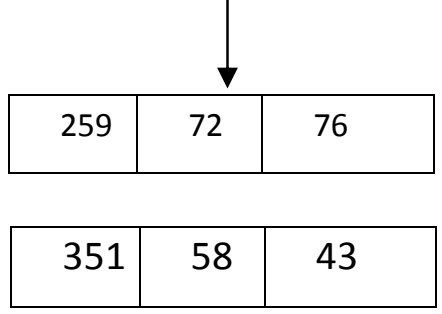

The newly obtained chromosomes from crossover operation are then pushed for mutation. By performing mutation, a new chromosome will be generated as illustrated below.

Before Mutation

\begin{tabular}{|c|c|c|}
\multicolumn{1}{c}{} & $\downarrow$ & $\downarrow$ \\
\hline 259 & 72 & 76 \\
\hline
\end{tabular}

After Mutation

\begin{tabular}{|l|l|l|}
\hline 259 & 76 & 72 \\
\hline
\end{tabular}

This is done by random generation of two points and then performing swaps between both the genes.

\section{(ii) Objective function}

The objective function that enumerates the optimality of the solution in a genetic Algorithm is known as fitness function. The fitness function is taken from Radhakrishnan et al (2010) and is given as:

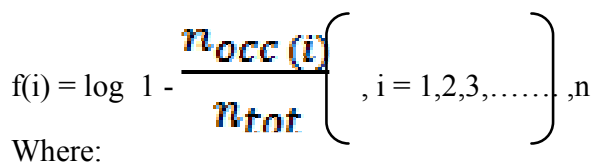

$n_{\text {ocC }}(i)=$ The Average of the number of occurrences of the stock of manufacturing unit, distributions centers 1 and 2 within the range of +10 and -10 in 52 weeks in the record set.

$n_{\text {tot }}=$ The total number of data present, i.e. 52 , in the record set.

But in that paper (Radhakrishnan et al, 2010), they have just calculated the optimal stock levels at different levels of supply chain. But, in this paper, we intend to calculate the ROL of the factory in a web enabled environment.

(iii) Reorder Level

The objective is of minimization type and the value of $\mathrm{f}(\mathrm{i})$ always lies between -1 and 0 . Since we have taken the range within \pm 10 , the reorder level will be increased to 10 if the value of objective function lies between -0.5 to 0 and the reorder level will be decreased by 10 if the value of $\mathrm{f}(\mathrm{i})$ lies between -1 to -0.5 .

\section{IMPLEMENTATION OF WEB ENABLED INVENTORY OPTIMIZATION}

The web implementation comprises the article module, factory module, distribution center 1 module, distribution center 2 modules and genetic algorithm module. The brief description of the modules is given below:

(i) Article Module:

The Article Module contains the "new registration" and "article view". The New registration is used to include the new article having the fields Article Id, Category, Type, color, Size, MRP and ROL. The article view is used to 
display the master view of the article, i.e., existing article list having the field name as article Id, category, Type, Color, Size, MRP and ROL.

\section{(ii) Factory Module:}

The Factory Module consists of the Stock Entry and Stock Position. The stock entry is used to enter the closing stock of the week of a particular article Id as shown in Figure 2.

Factory stock position with respect to the Article id is displayed with the fields article Id, category, type, Color, Size, Weeks number and Stock position as shown in Figure 3.

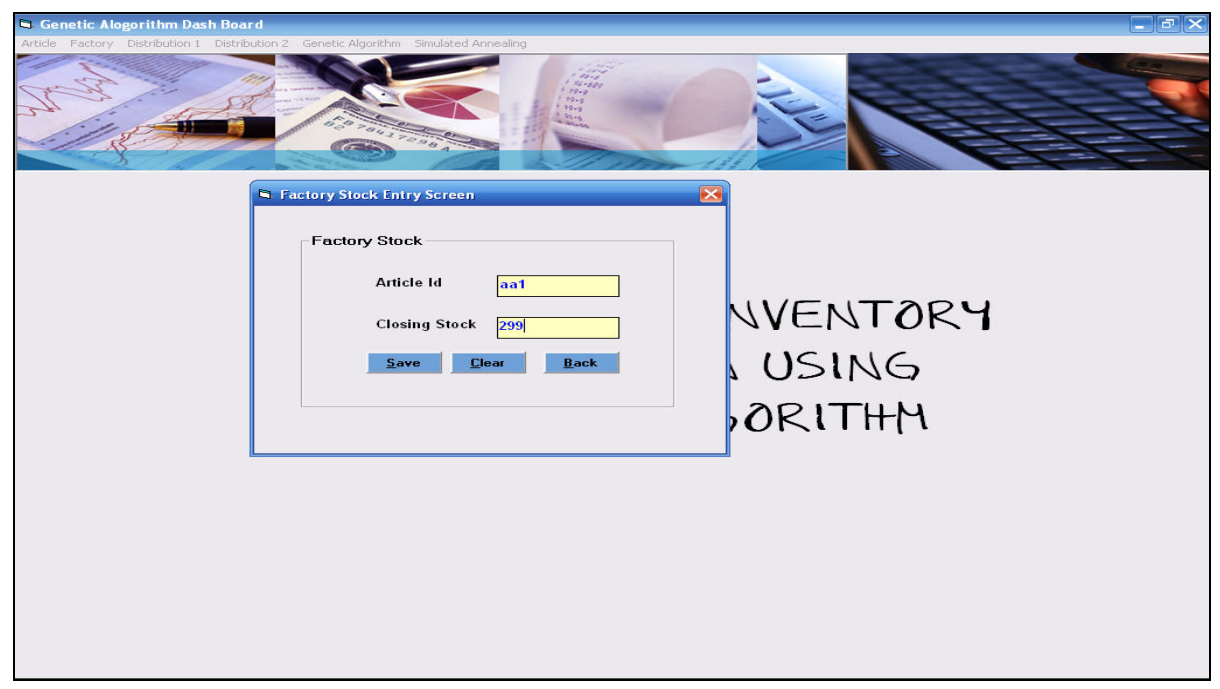

Figure 2 Factory stock entry screen

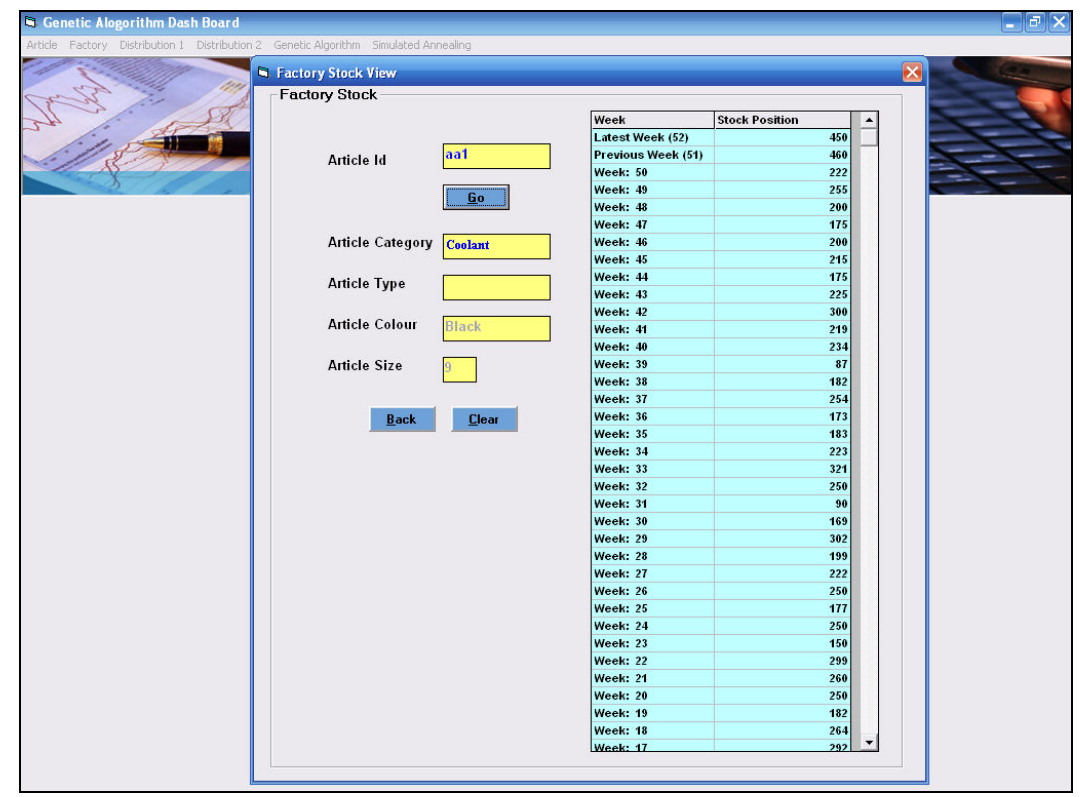

Figure 3 Factory stock position view

(iii) Distribution Center 1 and Distribution Center 2 Module

The Distribution Centers module contains the two categories similar to that as the factory Module, Stock and Stock Position. The closing stock of the particular week of the respective distribution centers with article Id is entered.
Distribution Center stock position with respect to the article Id is displayed with the field's article Id, category, type, Color, Size, Weeks number and Stock position. 


\section{(iv) Genetic Algorithm Module}

The Genetic Algorithm module contains three sub-modules Chromosome Representation, Crossover and Mutation. Chromosome representation consists of Chromosome I and Chromosome II with the article Id as shown in Figure 4.

The fitness function of chromosome I can be found. The crossover module performs the crossover operation and displays the new Chromosome with the respective fitness function as shown in Figure 5.

The mutation Module performs the mutation and gives the new chromosome with the fitness function value with new optimized ROL as shown in Figure 6.

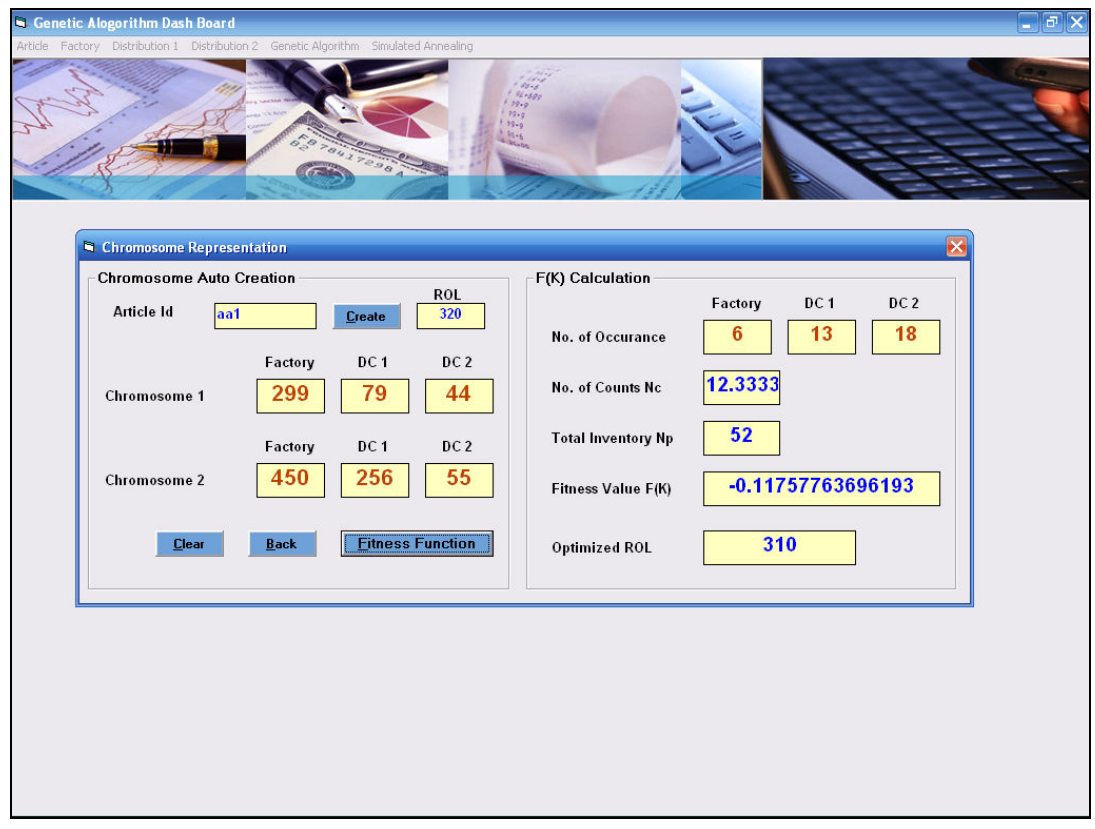

Figure 4 Chromosome representation

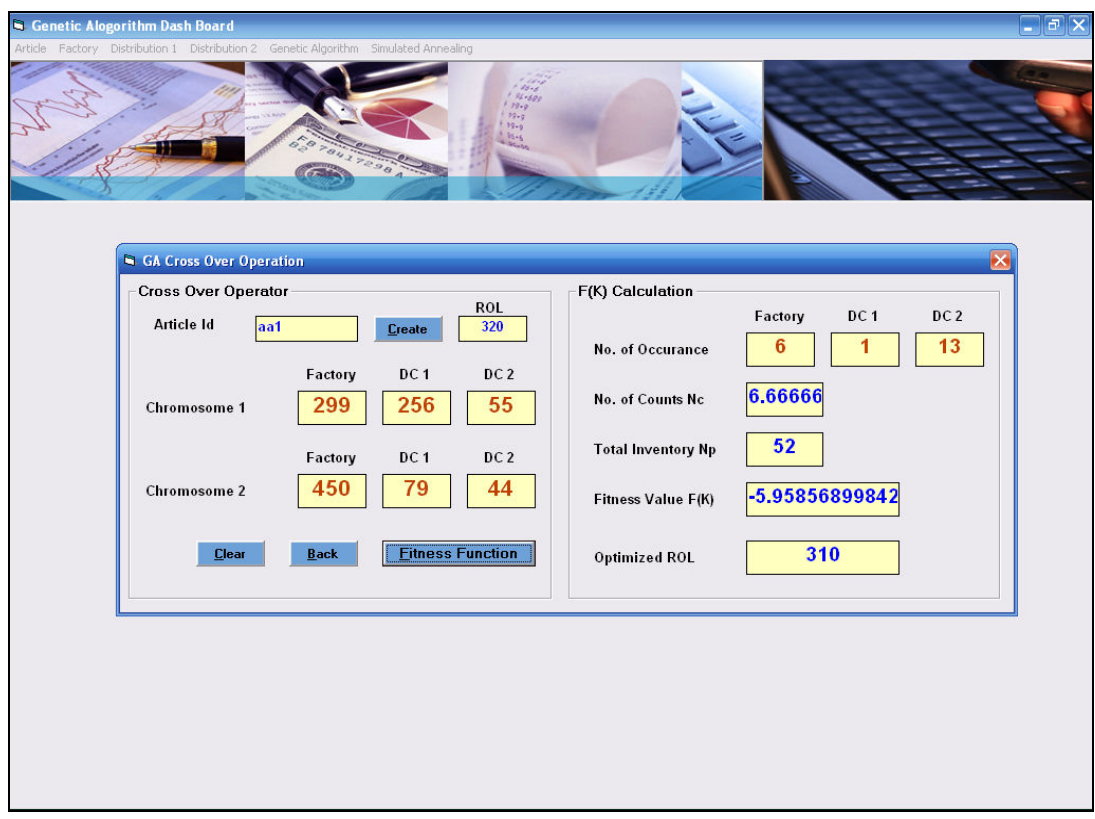

Figure 5 Illustration of cross over operation 


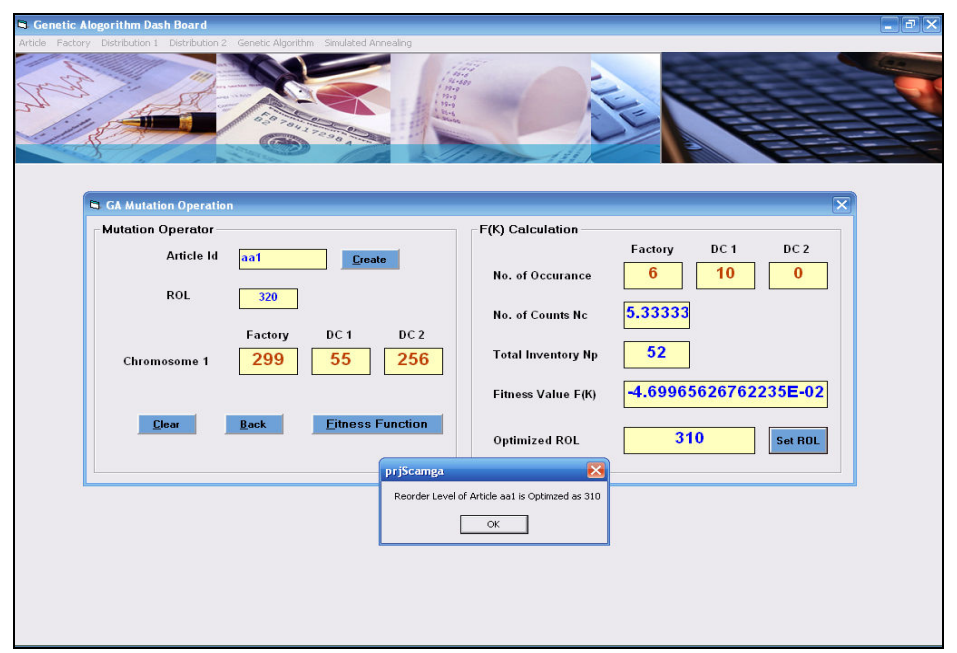

Figure 6 Illustration of mutation operation

Thus the developed model facilitates to optimize the reorder level each week in the automobile manufacturing unit.

\section{CONCLUSIONS}

Inventory is a major source of cost in a supply chain and has huge impact on responsiveness, so inventory management becomes significant entity of supply chain management. This paper has demonstrated the optimization of the ROL in manufacturing industry, wholesaler and retailer, which in turn needs the information of the stock level and the needed ordering quantities of the multiple products. A novel approach based on genetic algorithm is applied to determine the most probable ROL required for inventory optimization in the supply chain which ensures minimization of the total supply chain cost. The proposed future work is to apply the method for increased number of distribution centers and to implement other heuristics such as simulated annealing for the same.

\section{REFERENCES}

[1] Beamon, B.M., "Supply Chain design and Analysis: Models and Methods", International Journal of Production Economics, Vol. 55, pp: 281-294, 1998.

[2] Chandra K. Jaggi, Aggarwal. K.K and Priyanka Verma, "Two Warehouse Inventory Model For Deteriorating Items When Demand Is Price Sensitive", International Journal of Operational Research, Vol. 7,No. 4, Pg 530 - 543, 2010.

[3] Gunasekaran. A, Patel. C, Ronald E. McGaughey, “A Framework For Supply Chain Performance Measurement", International Journal of Production Economics, Vol. 87, pp: 333 - 347, 2004.

[4] Jeffery Joines, Gupta, Mahmut Ali Gokee, Michael G. Kay, Russell E. King, "Supply Chain Multi Objective Optimization", Proceeding of the 2002 Winter Simulation Conference, 2002.

[5] Jianhui Wang, Lin Xu, Shufang Sun and Yongliang Yan, "Optimization of Spare Parts Stores Based on an Improved Genetic Algorithm in a Supply Chain",
International Journal of Information and System Sciences, Vol. 2, No. 1, pp: 59-66, 2006.

[6] Kannan. G, Noorul Haq and Devika . M, "Analysis of Closed Loop Supply Chain using Genetic Algorithm and Particle Swarm Optimization", International Journal of Production Reearch, Vol. 47, pp 1175 1200, 2009.

[7] Maiti. M and Maity. K, "Optimal Inventory Policies For Deteriorating Complementary And Substitute Items", International Journal of System Science, Vol. 40, pp:. $267-276,2009$.

[8] Narmadha. S, Selladurai. V, Sathish. G, "Multiproduct Inventory Optimization using Uniform Cross Over Genetic algorithm", IJCSIS, Vol. 7, No. 1, 2010.

[9] Narmadha. S, Selladurai. V, Sathish. G, "Efficient Inventory Optimization of multiproduct, multiple suppliers with lead time using PSO", IJCSIS, Vol. 7, No. 1, 2010.

[10] Radhakrishnan. P, Prasad V.M. and Jeyanthi. N, "Predictive analysis using Genetic Algorithm for Efficient Supply Chain Management", Journal of Computer Science and Network Security", Vol. 10, No. 3, pp:. 1820-0186., 2010.

[11] Radhakrishnan. P, Prasad. V.M and Jeyanthi. N, "Design of Genetic Algorithm based Supply chain Inventory Optimization with Lead Time", Journal of Computer Science and Network Security", Vol. 10, No. 3, pp: 1820-1826, 2010.

[12] Sean X. Zhou and Xiuli Chao, "Optimal Policy for a Multi Echelon Inventory system with Batch Ordering and Fixed Replenishment Intervals" , Operations Research, Vol. 57, pp: 377-390, 2009.

[13] Uthayakumar. R and Geetha. K.V, "Replenishment Policy For Single Item Inventory Model With Money Inflation”, Opsearch, Vol. 46, pp: 345-357, 2008. 